\title{
Spin-wave excitations in ribbon-shaped Fe nanoparticles
}

\author{
P. Crespo, ${ }^{1,2}$ J. M. González, ${ }^{1,3}$ A. Hernando, ${ }^{1,2}$ and F. J. Yndurain ${ }^{4}$ \\ ${ }^{1}$ Instituto de Magnetismo Aplicado. UCM, RENFE, CSIC. Las Rozas P.O. Box 155, 28230 Madrid, Spain \\ ${ }^{2}$ Dpto. de Física de Materiales, UCM, Madrid, Spain \\ ${ }^{3}$ Instituto de Ciencia de Materiales, CSIC, Cantoblanco, Madrid, Spain \\ ${ }^{4}$ Dpto. de Física Teórica, UAM, Cantoblanco, Madrid, Spain \\ (Received 29 September 2003; published 15 January 2004)
}

\begin{abstract}
It has been found that in highly anisometric ribbon-shaped Fe particles with nanoscale dimensions that the magnetization decreases with temperature markedly faster than in bulk bcc Fe. This anomalous dependence, which becomes more remarkable as the length-to-cross section ratio increases, arises from the elongated shape of the particles. The analytical approximation performed on the thermal spectrum of magnons, compatible with the sample dimensions, unravels the correlated influences of shape and size on the thermal decreasing rate of magnetization.
\end{abstract}

It has been observed that some magnetic properties considered as intrinsic when measured in the bulk exhibit an anomalous behavior when the size of magnetic entities decreases down to the nanoscale. ${ }^{1}$ In particular, the thermal dependence of the magnetization deviates from that expected from the Bloch law. ${ }^{2}$ Since in very small size particles magnons with wavelengths larger than the particle dimensions cannot be excited, a threshold of thermal energy is required to create spin waves. In this paper we present a nanoscale effect linked to the shape of very small particles. Opposite to the consequences of the known size effect, ${ }^{2}$ which essentially contributes to stabilizing the magnetization, the shape effect analyzed here leads, in the low temperature range, to a decrease of the magnetization with temperature which is faster than that measured in bulk samples.

The experimental magnetic behavior of wire shaped $\mathrm{Cu}-15$ vol \% Fe composite alloys, produced by the heavy working of spray-deposited billets, has been analyzed. A detailed description of material fabrication has been reported elsewhere. ${ }^{3}$ With the cold working proceeding the spheroidal Fe particles, about $1 \mu \mathrm{m}$ in size, become drawn into elongated, flattened ribbons oriented along $\langle 110\rangle$ direction with progressively smaller cross sections. Due to the high and positive enthalpy of mixing that characterizes the $\mathrm{Fe}-\mathrm{Cu}$ system it is expected that $\mathrm{Fe}$ and $\mathrm{Cu}$ have not reacted, forming a $\mathrm{FeCu}$ solid solution. ${ }^{4}$ Transmission electron microscopy shows that the thickness of the Fe ribbons decreases with the increase of the drawing strain from $14 \mathrm{~nm}$ down to $3.5 \mathrm{~nm}$, while the ribbons width drops from $260 \mathrm{~nm}$ down to $27 \mathrm{~nm}$. Hereinafter, the studied samples will be identified by their drawing strain measured by the wire diameter after drawing, $\Phi$.

A magnetic characterization below room temperature was carried out by means of a superconducting quantum interference magnetometer, under a maximum applied field of $5 \mathrm{~T}$ strong enough to saturate the samples. Figure 1 shows the thermal dependence of magnetization $M$, measured in all the composite samples. Data corresponding to bulk bcc Fe were also included in the figure.

It should be remarked from Fig. 1 that the magnetization of the composites decreases with $T$ much faster than in bulk
Fe. In addition, as the length-to-cross section ratio increases the magnetization decreasing rate enhances and deviates from the typical $T^{3 / 2}$ dependence. For small enough crosssectional dimensions (i.e., wire diameters of 0.12 and 0.26 $\mathrm{mm}$ ) a rapid and almost linear decrease with temperature is observed.

Since the only difference between the samples is related to the measured cross section (and consequently the elongation) of bcc Fe nanoribbons, the deviation from the expected behavior could only be attributed to shape effects. Within a spin wave framework the thermal dependence of magnetization is related to the total number of excited magnons per unit volume, $\Sigma_{k} n_{\mathbf{k}}$, as follows:

$$
M(T)=M(0 K)\left[1-\frac{1}{N S} \sum_{\mathbf{k}} n_{\mathbf{k}}\right],
$$

where $N$ is the number of atoms with spin $S$ per unit volume and $\mathbf{k}$ is the magnon wave vector.

Relation (1) leads in bulk materials to the well known Bloch $T^{3 / 2}$ law. The energy of an excitation with wave vector

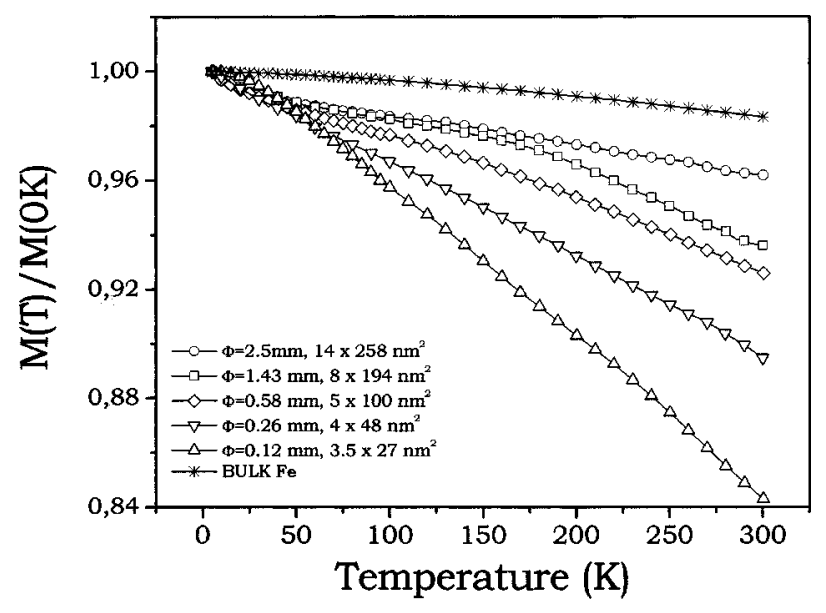

FIG. 1. Temperature dependence of the magnetization after different drawing strains, i.e., wire diameters $\Phi$. The cross section dimensions for the bcc-Fe ribbons are also indicated. The evolution of bulk bcc $\mathrm{Fe}$ is also shown for comparison 


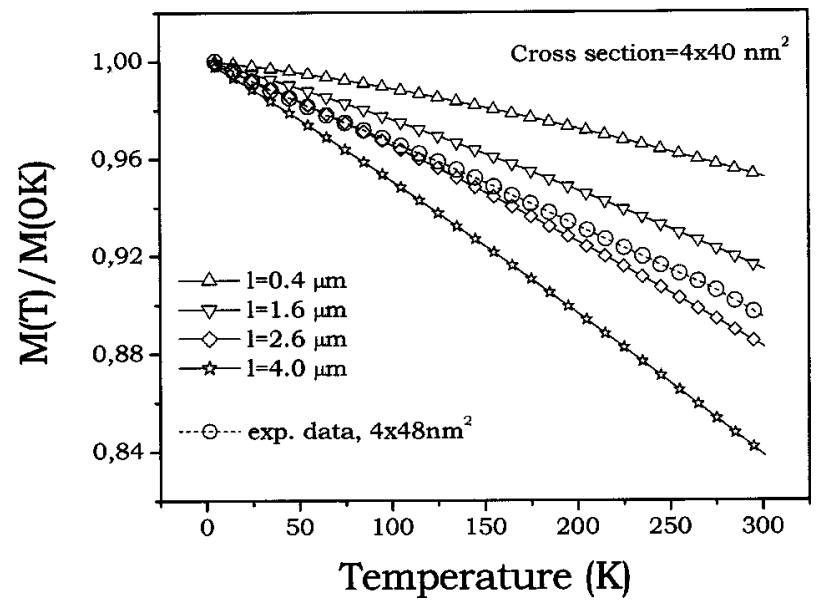

FIG. 2. Calculated curves for nanoribbons with $4 \times 40-\mathrm{nm}^{2}$ cross section and different lengths. The experimental data of the $\phi=0.26 \mathrm{~mm}$ sample $\left(4 \times 48-\mathrm{nm}^{2}\right.$ cross section $)$ have also been plotted. The ribbon length spreads over a broad range but an average value of $2 \mu \mathrm{m}$ could account for the observed behavior according to the ribbon length dependence shown in the figure.

k can be written as $\varepsilon=D k^{2}$, where $D$ is the exchange stiffness constant that takes a value of $D=0,281 \mathrm{eV} \AA^{2}$ for bcc Fe. ${ }^{5}$ Note that in a ribbon with length $l$, width $w$ and thickness $t$ the incompatibility of the large wavelength, $\lambda$, spin waves with the reduced system dimensions leads to the following dependence of the number of magnons on $\lambda$ : For $l$ $>\lambda>w, \mathbf{k}$ is constrained to be a vector lying along the longitudinal direction. For $w>\lambda>t$, $\mathbf{k}$ behaves as a twodimensional (2D) vector lying in the plane defined by $l$ and $w$. Finally, for $t>\lambda, \mathbf{k}$ becomes a $3 \mathrm{D}$ vector similar to the bulk material case. After taking into consideration the corresponding $k$ densities it can be easily found that the number of magnons per unit volume can be written as a function of $T$ according to

$$
\begin{aligned}
& \sum_{\mathbf{k}} n_{\mathbf{k}}=\frac{1}{l w t}\left[\frac{l}{2 \pi} \int_{2 \pi / l}^{2 \pi / w} \frac{d k}{\left.\exp \left(\frac{D k^{2}}{k_{B} T}\right)-1\right]}\right. \\
& +\frac{l w}{2 \pi} \int_{2 \pi / w}^{2 \pi / t} \frac{k d k}{\left.\exp \left(\frac{D k^{2}}{k_{B} T}\right)-1\right]} \\
& \left.+\frac{l w t}{2 \pi^{2}} \int_{2 \pi / t}^{\infty} \frac{k^{2} d k}{\left[\exp \left(\frac{D k^{2}}{k_{B} T}\right)-1\right]}\right],
\end{aligned}
$$

where $k_{B}$ is the Boltzmann constant. Note that in bulk materials only the last integral of the right hand side holds and when the integration limits are 0 and infinity it leads directly to the Bloch law.

From Eqs. (1) and (2) it is possible to reproduce the experimental curves as shown in Fig. 2 in which the calculated effect of the aspect ratio on the thermal dependence of magnetization is illustrated for nano-ribbons with $4 \times 40-\mathrm{nm}^{2}$ cross section and different lengths. The experimental curve corresponding to the sample containing nanoribbons with similar cross section has also been plotted in the figure. The average ribbon length $l$, that can not be obtained by TEM, has been estimated from the fitting as being close to $2 \mu \mathrm{m}$.

It is remarkable the influence of shape on the thermal dependence of magnetization. Indeed, as pointed out by the results shown in Fig. 2, the elongated shape is responsible for the extremely fast magnetization decrease observed in samples submitted to the largest drawing strains (see Fig. 1). In order to discuss conditions required for the observation of this shape effect let us analyze the thermal dependence of magnetization as a function of the elongation of the particle. For the sake of simplicity it will be assumed that $t=w$ and $l=h t$, where $h$ is the aspect ratio of the particle. In this case the second integral in Eq. (2) vanishes and the number of magnons per unit volume becomes

$$
\sum_{\mathbf{k}} n_{\mathbf{k}}=\frac{1}{2 \pi w t} I_{1}(h, t, T)+\frac{1}{2 \pi^{2}} I_{3}(t, T) .
$$

Considering $t>\left(2 \pi D / k_{B} T\right)^{1 / 2}$, a condition that for a ribbon with $t=100 \AA$ holds at $T=2.1 \mathrm{~K}$, the total number of magnons per unit volume given by Eq. (3) can then be written as (see Appendixes A and B)

$$
\sum_{\mathbf{k}} n_{\mathbf{k}}=A \frac{(h-1)}{t} T+B T^{3 / 2}-C \frac{T^{5 / 4}}{t^{1 / 2}},
$$

where

$$
A=\left[1 /(2 \pi)^{2}\right] k_{B} / D, \text { and } B=\left(k_{B} / 4 \pi D\right)^{3 / 2} \zeta(3 / 2),
$$

$\zeta$ being the Riemann zeta function and

$$
C=\left[1 /\left(2 \pi^{3}\right)^{1 / 2}\right]\left(k_{B} / D\right)^{5 / 4} .
$$

For the conditions corresponding to our experimental data $t>\left(2 \pi D / k_{B} T\right)^{1 / 2}$ and, consequently, expression (4) holds as a good approximation. That expression (4) contains the well known term corresponding to the Bloch law for bulk materials, the $B T^{3 / 2}$ term, and two additional terms, with coefficients $A$ and $C$, linked to the small size of the particles and vanishing for large size systems (increasing $t$ values). The opposite sign as well as the different temperature dependence of both terms enable to distinguish between size, $t$, and shape, $h$, effects, as illustrated in Fig. 3 . The positive and linear with $T$ first term, including coefficient $A$, contributes to enhance the number of excited magnons and describes the shape effect through its $h$ dependence, though also decreases with the inverse of the size. In particular, the extra number of magnons per unit volume originated by the first term at 300 $\mathrm{K}$ is given by $0.0023((h-1) / t[\AA])$. Consequently, the influence of the shape effect for particles with $t=10^{4} \AA(1 \mu \mathrm{m})$ requires to be relevant an aspect ratio $h$ as large as $10^{5}$. The negative term with $C$ coefficient varies as $T^{5 / 4}$ and only depends on size and, oppositely to the first one, decreases as $t$ does. Therefore, the shape effect that gives rise to a fast and linear decrease of $M$ with $T$, shown in Fig. 1, is only relevant for small particle sizes. But even at this range of small $t$ the first term influence decreases as the spherical shape is approached, i.e., $h-1 \ll t[\AA]$. Notice that for $h=1$, only the 


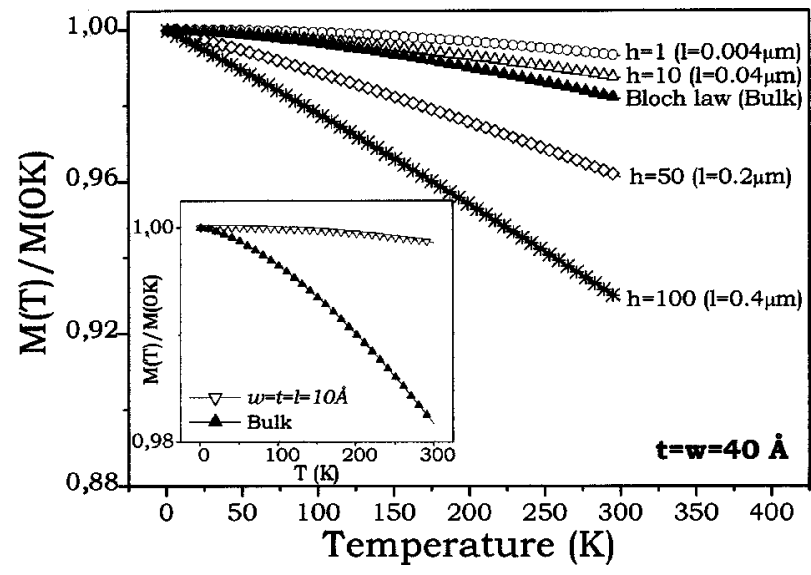

FIG. 3. Thermal dependence of magnetization given by expression (4) for a sample with $t=w=40 \AA$ and different aspect ratios, $h$. The inset shows the temperature dependence of the magnetization in the low $T$ and/or $t$ limit for $h=1$. The thermal dependence for a bulk sample (i.e., Bloch law) is also included.

second term in Eq. (3) contributes to $\Sigma_{k} n_{\mathbf{k}}$ and when $t$ decreases below $\left(2 \pi D / k_{B} T\right)^{1 / 2}$, the high temperature approximation considered in Eq. (4) is not valid and, as shown in the Appendix (B), $I_{3}$ can then be approximated as

$$
I_{3}=\frac{1}{2 a^{3 / 2}}\left\{\sqrt{\hat{\varepsilon}} e^{-\hat{\varepsilon}}-\sqrt{\hat{L}} e^{-\hat{L}}\right\},
$$

with $\hat{\varepsilon}=\left(D / k_{B} T\right)^{1 / 2}(2 \pi / t)$ and $\hat{L}=\left(D / k_{B} T\right)^{1 / 2}\left(2 \pi / a^{*}\right), a^{*}$ being the lattice constant of bcc Fe. It is obvious that the negative term including $C$ coefficient is responsible for $h$ $=1$ of the cut-off frequency predicted for magnons in very small systems. ${ }^{2}$ This effect is illustrated in the inset of Fig. 3, where the magnetization calculated from Eq. (5) for a particle with $t=10 \AA$ and $h=1$ is shown.

In summary, the experimental data on the thermal dependence of magnetization of elongated $\mathrm{Fe}$ nanoribbons has been analyzed in terms of a spin wave analytical model based on the compatibility between the excitable magnon wavelength and the particle dimensions. The analytical expression given by Eq. (4) for the number of magnons per unit volume, valid at the high temperature range, enables one to directly understand the influence of shape and size on the equilibrium thermal excitation. This expression encloses the standard Bloch term and two additional ones associated with sample morphology and dimensions. Both terms are only relevant for small dimensions, i.e., small $t$; but, whereas the positive shape effect term contributes to increase the thermal decreasing rate of magnetization the negative size effect term tends to delay this thermal decrease. The shape effect appears as a consequence of the structure of the $I_{1}$ integrand in Eq. (2) that diverges for any $T$ different of zero as $k$ approximates zero. Therefore, the shape effect term in Eq. (4) can be understood by considering that the contribution of $I_{1}$ to the total number of magnons sharply increases as the lower integration limit decreases (i.e., as $h$ increases) and the integration interval $(1 / t)[1-(1 / h)]$ increases (i.e., as $t$ decreases and $h$ increases).
The authors are indebted to Dr. D. G. Morris for sample preparation.

\section{APPENDIX A}

Let

$$
I_{1}(h, t, T)=\int_{2 \pi / l}^{2 \pi / w} \frac{d k}{\left[\exp \left(\frac{D k^{2}}{k_{B} T}\right)-1\right]},
$$

that can be written as

$$
I_{1}(\varepsilon)=\int_{\varepsilon}^{L} \frac{d k}{e^{a k^{2}}-1},
$$

where

$$
\varepsilon=\frac{2 \pi}{l}=\frac{2 \pi}{h t}, \quad L=\frac{2 \pi}{w}=\frac{2 \pi}{t} ; a=\frac{D}{k_{B} T} .
$$

For $a L^{2} \leqslant 2 \pi$ (high $T$ and/or high $t$ limits) the integral becomes

$$
I_{1}(\varepsilon)=\sum \frac{B_{j}}{j ! a} a^{j} \int_{\varepsilon}^{L} d k k^{2 j-2},
$$

where $B_{j}$ are the Bernouilli numbers. Therefore, one obtains

$$
\begin{aligned}
I_{1}(\varepsilon) \cong & \frac{1}{a}\left(\frac{1}{\varepsilon}-\frac{1}{L}\right)-\left(\frac{L-\varepsilon}{2}\right)+\frac{a}{36}\left[L^{3}-\varepsilon^{3}\right]+O\left(\frac{a^{3} L^{7}}{(2 \pi)^{3}}\right) \\
& +O\left(\frac{a^{3} \varepsilon^{7}}{(2 \pi)^{3}}\right),
\end{aligned}
$$

that in first order becomes

$$
I_{1}(h, t, T) \cong \frac{k_{B} T}{2 \pi D} t(h-1) .
$$

\section{APPENDIX B}

Let

$$
I_{3}(t, T)=\int_{2 \pi / t}^{\infty} \frac{k^{2} d k}{\left[\exp \left(\frac{D k^{2}}{k_{B} T}\right)-1\right]}
$$

or

$$
I_{3}(\varepsilon)=\int_{\varepsilon}^{L} \frac{k^{2} d k}{e^{a k^{2}}-1}
$$

where $\varepsilon=2 \pi / t, L$ is a high enough number and $a$ $=D / k_{B} T$, and

$$
I_{3}(\varepsilon)=\int_{\varepsilon}^{L} \frac{k^{2} d k}{e^{a k^{2}}-1} \equiv \frac{1}{2 a^{3 / 2}} \hat{I}_{3},
$$

where 


$$
\hat{I}_{3}=\int_{\hat{\varepsilon}}^{\hat{L}} d x \frac{x^{1 / 2}}{e^{x}-1}
$$

and $\hat{\varepsilon}=a^{1 / 2} \varepsilon$ and $\hat{L}=a^{1 / 2} L$

The latter can be written as

$$
\begin{aligned}
\hat{I}_{3} & =\left\{\int_{0}^{\infty} d x \frac{x^{1 / 2}}{e^{x}-1}-\int_{0}^{\hat{\varepsilon}} d x \frac{x^{1 / 2}}{e^{x}-1}-\int_{\hat{L}}^{\infty} d x \frac{x^{1 / 2}}{e^{x}-1}\right\} \\
& =\left.\hat{I}_{3}\right|_{0} ^{\infty}-\left.\hat{I}_{3}\right|_{0} ^{\hat{\varepsilon}}-\left.\hat{I}_{3}\right|_{\hat{L}} ^{\infty}
\end{aligned}
$$

and

$$
\left.\hat{I}_{3}\right|_{0} ^{\infty}=\int_{0}^{\infty} d x \frac{x^{1 / 2}}{e^{x}-1}=\frac{\sqrt{\pi}}{2} \zeta\left(\frac{3}{2}\right),
$$

where $\zeta\left(\frac{3}{2}\right)$ is the Riemann zeta function,

$$
\left.\hat{I}_{3}\right|_{\hat{L}} ^{\infty}=\int_{\hat{L}}^{\infty} d x \frac{x^{1 / 2}}{e^{x}-1} \cong \sqrt{\hat{L}} e^{-\hat{L}}\{1+O(1 / \hat{L})\} .
$$

As concerns $\left.\hat{I}_{3}\right|_{0} ^{\hat{\varepsilon}}$, two different limits should be considered.

(i) For $\hat{\varepsilon}$ small enough with respect to $2 \pi,\left.\hat{I}_{3}\right|_{0} ^{\hat{\varepsilon}}$ can be approximated as follows:

$$
\left.\hat{I}_{3}\right|_{0} ^{\hat{\varepsilon}}=\int_{0}^{\hat{\varepsilon}} d x \frac{x^{1 / 2}}{e^{x}-1}=\sqrt{\hat{\varepsilon}}\left\{2-\frac{\hat{\varepsilon}}{3}+\frac{\hat{\varepsilon}^{2}}{30}-\frac{\hat{\varepsilon}^{4}}{3240}+\cdots\right\} .
$$

Therefore

$$
\begin{aligned}
I_{3}= & \frac{1}{2 a^{3 / 2}} \hat{I}_{3} \\
= & \frac{1}{2 a^{3 / 2}}\left\{\frac{\sqrt{\pi}}{2} \zeta\left(\frac{3}{2}\right)-\sqrt{\hat{\varepsilon}}\left\{2-\frac{\hat{\varepsilon}}{3}+\frac{\hat{\varepsilon}^{2}}{30}-\frac{\hat{\varepsilon}^{4}}{3240}+\cdots\right\}\right. \\
& \left.-\sqrt{\hat{L}} e^{-\hat{L}_{\{1}}\{(1 / \hat{L})\}\right\},
\end{aligned}
$$

or, in first order, $\left.I_{3}(t, T)=\sqrt{\pi} \zeta(3 / 2) / 2\right]\left(k_{B} T / D\right)^{3 / 2}$ $-(2 \pi / t)^{1 / 2}\left(k_{B} T / D\right)^{5 / 4}$.

(ii) For $\hat{\varepsilon}$ high enough with respect to unity, $\left.\hat{I}_{3}\right|_{0} ^{\hat{\varepsilon}}$ should be written as follows:

$$
\begin{aligned}
\hat{I}_{3} \mid \hat{\varepsilon} & =\int_{0}^{\hat{\varepsilon}} d x \frac{x^{1 / 2}}{e^{x}-1}=\int_{0}^{\hat{\varepsilon}} d x x^{1 / 2} e^{-x} \sum_{n=0}^{\infty} e^{-n x} \\
& =\sum_{n=1}^{\infty} \frac{\gamma(3 / 2, n \hat{\varepsilon})}{n^{3 / 2}},
\end{aligned}
$$

where $\gamma(a, z)$ is the incomplete gamma function. Therefore, $\hat{I}_{3}$ can be written as follows:

$$
\begin{aligned}
\hat{I}_{3}= & \sqrt{\hat{\varepsilon}} \sum_{n=1}^{\infty} \frac{e^{-n \hat{\varepsilon}}}{n}\left\{1+\frac{1}{2 n \hat{\varepsilon}}-\frac{1}{4 n^{2} \hat{\varepsilon}^{2}}\right. \\
& \left.-\frac{1}{2 \sqrt{\pi}} \sum_{j=3}^{\infty}(-1)^{j} \frac{\Gamma(j-1 / 2)}{n^{j} \hat{\varepsilon}^{j}}\right\}-\sqrt{\hat{L}} e^{-\hat{L}}\{1+O(1 / \hat{L})\} .
\end{aligned}
$$

By neglecting the addition over $j$ and taking $n=1,2,3 \ldots$ we finally obtain

$$
\begin{aligned}
I_{3}= & \frac{1}{2 a^{3 / 2}} \hat{I}_{3}=\frac{1}{2 a^{3 / 2}}\left\{\sqrt { \hat { \varepsilon } } e ^ { - \hat { \varepsilon } } \left\{1+\frac{1}{2 \hat{\varepsilon}}-\frac{1}{4 \hat{\varepsilon}^{2}}\right.\right. \\
& \left.+\frac{e^{-\hat{\varepsilon}}}{2}\left(1+\frac{1}{4 \hat{\varepsilon}}-\frac{1}{16 \hat{\varepsilon}^{2}}\right)+\frac{e^{-2 \hat{\varepsilon}}}{3}\left(1+\frac{1}{6 \hat{\varepsilon}}-\frac{1}{36 \hat{\varepsilon}^{2}}\right)\right\} \\
& \left.-\sqrt{\hat{L}} e^{-\hat{L}}\{1+O(1 / \hat{L})\}\right\} .
\end{aligned}
$$

${ }^{1}$ J. F. Loffler, H. B. Braun, and W. Wagner, Phys. Rev. Lett. 85, 1990 (2000); A. Hernando and T. Kulik, Phys. Rev. B 49, 7064 (1994).

${ }^{2}$ N. García, in Science and Technology of Nanostructured Magnetic Materials, Vol. 259 of NATO Advanced Study Institute Series, Series B: Physics, edited by G. C. Hadjipanayis and G. A. Prinz (Plenum, 1990), p. 581; V. L. Safonov and H. Neal Bertram, Phys. Rev. B 63, 094419 (2001); C. Mathieu, J. Jorzik, A. Frank, S. O. Demokritov, A. N. Slavin, B. Hillebrands, B. Bar- telian, C. Chappert, D. Decanini, F. Rousseaux, and E. Cambril, Phys. Rev. Lett. 81, 3968 (1998).

${ }^{3}$ C. Biselli and D. G. Morris, Adv. Mater. (Weinheim, Ger.) 44, 493 (1996).

${ }^{4}$ A. Hernando, P. Crespo, J. M. Barandiarán, and A. García Escorial, Phys. Rev. Lett. 70, 3521 (1993).

${ }^{5}$ B. Antonini, F. Menzinger, A. Paoletti, and A. Tucciarone, Phys. Rev. 178, 833 (1969). 